\title{
Prevalencia del Síndrome de Burnout académico en el estudiantado de Enfermería de la Universidad de Costa Rica ${ }^{1}$
}

\author{
Nataly Bolaños Reyes ${ }^{2}$ \\ Nazareth Rodríguez Blanco ${ }^{3}$
}

Institución: Universidad de Costa Rica

\section{COMO CITAR}

Bolaños, N. y Rodríguez, N. (2016). Prevalencia del Síndrome de Burnout académico en el estudiantado de Enfermería de la Universidad de Costa Rica. Rev. Enfermería Actual en Costa Rica, 31, 1-19. DOI: http://dx.doi.org/10.15517/revenf.v0i31.24519

\section{RESUMEN}

Introducción. El síndrome de Burnout se suele investigar más en el área laboral, sin embargo quienes trabajan no son los únicos individuos que sufren de este síndrome, también muchos estudiantes se encuentran inmersos en esta situación, debido a la carga tanto académica como personal que requiere cumplir con todos los requisitos universitarios.

Metodología. La población está formada por el estudiantado de la carrera de Licenciatura en Enfermería, de Escuela de Enfermería de la Universidad de Costa Rica. El diseño metodológico es cuantitativo, no experimental, con alcance descriptivo transeccional.

Resultado. el 18,8\% de la población estudiada presenta el sindromé de Burnout académico

Conclusión. la mayoría de la población presenta síntomas físicos como dolores de cabeza, trastornos del sueño y problemas osteomusculares. Además de síntomas conductuales como irritabilidad, nerviosismo, sentimientos de vacío y soledad, y síntomas cognoscitivos, como dificultad para concentrarse y mayor sensibilidad a la crítica que a la larga pueden ser el camino abierto a otros padecimientos tanto físicos como psiquiátricos.

Palabras clave: burnout-académico, estrés-académico, estudiantes-de-enfermería.

\footnotetext{
${ }^{1}$ Fecha de recepción: 15 de julio 2015

Fecha aceptación: 29 de noviembre 2015

${ }^{2}$ Enfermera. Trabajo independiente. Costa Rica. Correo electrónico: ntl bolare@hotmail.com

${ }^{3}$ Enfermera. Trabajo independiente. Costa Rica. Correo electrónico: nanardzblanko@,hotmail.com
} 


\title{
Burnout syndrome prevalence in academic nursing student at the University of Costa Rica ${ }^{1}$
}

Institution: University of Costa Rica

Nataly Bolaños Reyes ${ }^{2}$

Nazareth Rodríguez Blanco ${ }^{3}$

\section{CITED}

Bolaños, N. y Rodríguez, N. (2016). Burnout syndrome prevalence in academic nursing student at the University of Costa Rica. Rev. Enfermería Actual en Costa Rica, 31, 1-21. DOI: http://dx.doi.org/10.15517/revenf.v0i31.24519

\begin{abstract}
Introduction. The burnout syndrome is usually more researched in the workplace, but those working are not the only individuals who suffer from this syndrome, also many students are immersed in this situation, due to both academic load and personnel required to meet all university requirements.

Method. The population is made by the students of the Bachelor of Nursing, School of Nursing at the University of Costa Rica. The design methodology is quantitative, not experimental, with transeccional descriptive scope.

Results. $18.8 \%$ of the study population presents academic Burnout syndrome

Conclusion. Most of the population has physical symptoms such as headaches, sleep disorders and musculoskeletal problems. In addition to behavioral symptoms such as irritability, nervousness, feelings of emptiness and loneliness, and cognitive symptoms such as difficulty concentrating and increased sensitivity to criticism that eventually may be open to other ailments both physical and psychiatric way.
\end{abstract}

Key words: Burnout-academic, nursing- student, stress-academic

\footnotetext{
${ }^{1}$ Date of receipt: july 15, 2015

${ }^{2}$ Nurse. Independent work. Costa Rica. E-mail: ntl bolare@hotmail.com

${ }^{3}$ Nurse. Independent work. Costa Rica. E-mail: nanardzblanko@hotmail.com
}

Date of acceptance: November 29, 2015 


\title{
Prevalência da Síndrome de Burnout acadêmico nos estudantes de Enfermagem da Universidade de Costa Rica ${ }^{1}$
}

\author{
Nataly Bolaños Reyes ${ }^{2}$ \\ Nazareth Rodríguez Blanco ${ }^{3}$
}

Instituição: Universidade de Costa Rica

\section{Citação}

Bolaños, N. y Rodríguez, N. (2016). Prevalência da Síndrome de Burnout acadêmico nos estudantes de Enfermagem da Universidade de Costa Rica. Rev. Enfermería Actual en Costa Rica, 31, 1-21. DOI: http://dx.doi.org/10.15517/revenf.v0i31.24519

Introdução. A síndrome de Burnout é investigada mais na área trabalhista, no entanto, as pessoas que trabalham não são os únicos indivíduos que sofrem desta síndrome, também muitos estudantes se encontram imersos nesta situação, devido a carga tanto acadêmica como pessoal que requerem para cumprir com todos os requisitos universitários.

Método. A população está formada por estudantes da carreira de Licenciatura em Enfermagem, da Escola de Enfermagem da Universidade de Costa Rica. O desenho metodológico é quantitativo, não experimental, com alcance descritivo transeccional.

Resultado. $18,8 \%$ da população estudada apresenta a síndrome de Burnout acadêmico

Conclusão. A maioria da população apresenta sintomas físicos como dores de cabeça, trastornos do sono e problemas osteomusculares. Ademais dos sintomas de conduta como irritabilidade, nervosismo, sentimentos de vazio e solidão, e sintomas cognoscitivos, como dificuldade para se concentrar e maior sensibilidade a crítica que com o tempo, podem se tornar caminho aberto a outros padecimentos tanto físicos como psiquiátricos.

Palavras -chave: burnout-acadêmico, estresse-acadêmico, estudantes-de-enfermagem.

\footnotetext{
${ }^{1}$ Data de recepção: 15 de julho de 2015

Data de aceitação: 29 de novembro de 2015

${ }^{2}$ Enfermeira.Trabalho independente. Costa Rica. Correio eletrônico: ntl bolare@hotmail.com

${ }^{3}$ Enfermeira.Trabalho independente. Costa Rica. Correio eletrônico: nanardzblanko@hotmail.com
} 


\section{Revista Electrónica Enfermeria Actual en costa Rica}

\section{INTRODUCCIÓN}

El síndrome de burnout se suele investigar más en el área laboral, sin embargo los trabajadores no son los únicos individuos que sufren de este síndrome, ya que muchos estudiantes también se encuentran inmersos en esta situación, debido a la carga tanto académica como personal que requiere cumplir con todos los requisitos universitarios.

Hoy día se ha demostrado que el síndrome de burnout, que en un principio solo se investigaba en el ámbito laboral, ha extendido sus brazos hasta llegar al ámbito académico, principalmente en aquellas carreras en las que el estudiantado, por las características de estas, deben manejar altos niveles de estrés, debido a la alta carga académica.

El burnout organizacional o laboral es conceptualizado por Schaufeli, Martínez, Marques, Salanova y Bakker (2002) citado por Jácome, et al (2010,p. 38) como "un estado mental persistente, negativo, relacionado con el trabajo, que aparece en individuos normales y se caracteriza por agotamiento físico y emocional, acompañado de distrés, sentimientos de reducida competencia, poca motivación y actitudes disfuncionales en y hacia la actividad laboral", manifestaciones que son evidentes en los estudiantes de esta investigación, es decir, en el ámbito académico existe una vertiente que "surge de la presunción de que los estudiantes universitarios, al igual que cualquier profesional, se encuentran con presiones y sobrecargas propias de la labor académica" (Caballero, Domínguez, Hederich y Palacio, 2010, p. 133)

De igual forma, existe el burnout académico, definido como las "sensaciones en el estudiante de no poder dar más de sí mismo, una actitud cínica (o de distanciamiento, desinterés y pérdida del valor y sentido frente a las actividades académicas), y dudas acerca de la propia capacidad para realizarlo" (Jácome, et al, 2010, p. 38). Este síndrome genera consecuencias a nivel individual que afectarán más en un nivel u otro dependiendo de la circunstancia y de la persona. Al respecto, Existen investigaciones que "relacionan el síndrome de burnout y los problemas psicosomáticos, siendo la correlación más intensa con la sintomatología psicosomática del agotamiento emocional. Sin embargo, el diagnóstico médico como índice de falta de salud no se correlaciona con el síndrome de burnout" (Gonzales, Lacasta y Ordoñez, 2008, p.36).

Ahora bien, en el caso de los estudiantes, Barco, Miranda, Herrera y Caridad (2008) señalan que "el personal de enfermería tiene una alta predisposición a padecer el síndrome de burnout, sobre todo en la etapa de estudiante universitario", lo que indica que los estudiantes de enfermería tienen un alto riesgo de padecer este síndrome. Lo anterior es respaldado por Arango, et al. (2010), quienes determinan que el estrés ante los exámenes, ansiedad y depresión se relaciona con la aparición del síndrome.

En el ámbito costarricense este fenómeno ha sido poco estudiado: tan solo se conoce el trabajo de León (2013) indica, cómo los estudiantes, en su proceso de formación universitaria, pueden ser afectados por dicho síndrome, el cual se presenta como una manifestación del estrés crónico, producto de las condiciones psicológicas y sociales del proceso de formación. El autor observa que los estudiantes se ven afectados por cansancio emocional, relacionado con factores curriculares y sociales, generados por el estrés (sobrecarga académica, acceso a la tecnología, mediación docente en el aula, doble rol). 


\section{Revista Electrónica Enfermeria Actual en costa Rica}

Este (Burnout) se considera de gran importancia para la población estudiantil, porque se relaciona de manera directa con el quehacer del estudiante en su desempeño académico, modificando su conducta y la manera de afrontar las situaciones que se le presentan. La necesidad de investigar sobre este tema se justifica por la necesidad identificar si gran parte de la población sufre de un afrontamiento inadecuado del estrés, el cual le pueda llevar a sufrir de este síndrome.

Por último, se recalca la idea de que el síndrome de burnout académico implica un serio problema para la población estudiantil universitaria, pues disminuye su calidad como estudiante y, además, afecta a aquellos que se encuentran relacionados íntimamente a su proceso estudiantil.

\section{MATERIALES Y MÉTODO}

La población está formada por el estudiantado de la Licenciatura en Enfermería, de la Escuela de Enfermería de la Universidad de Costa Rica, activo y matriculado en cursos impartidos por la escuela de enfermería durante el segundo semestre correspondiente a cada año de carrera. Participaron un total de 289 Estudiantes, distribuidos por año de la siguiente forma: primer año: 72 , segundo año: 48 , tercer año: 52 , cuarto año: 57 y quinto año: 60 .

Esta investigación presenta un diseño metodológico cuantitativo no experimental con un alcance descriptivo puesto que busca finalmente, es transaccional porque los datos son recolectados en un solo momento. (Hernández, et al., 2010).

Para recolectar datos se empleó un cuestionario con ítems de tipo cerrado y semi-abierto, con respuestas únicas o múltiples. La primera parte del cuestionario se refiere a los aspectos sociodemográficos, económicos, familiares, educativos y personales, y consta de treinta preguntas; la segunda parte incluye el MBI-SS que mide el síndrome de burnout académico, el cual se divide en tres aspectos: el agotamiento emocional, medido a partir de cinco preguntas; el cinismo, con e cuatro preguntas y la autoeficacia académica, con cinco preguntas.

Al instrumento Maslach se le realizó un estudio estadístico de validez, mediante una comparación de las medidas entre las personas con puntuaciones más altas y más bajas obtenidas de los resultados de la prueba piloto con la muestra de 28 estudiantes, para cada ítem, con el propósito de conocer si efectivamente había diferencias entre las personas que contestaron. Se dice que si los individuos contestan siempre lo mismo en cada ítem este no es informativo, por lo que se determinó que el ítem 41 y 44 no discriminaban entre sujetos. El instrumento Maslach obtuvo un alfa de Cronbach de 0.929 lo que indica una calificación excelente para evaluar las variables del estudio.

La muestra de quienes contestaron el cuestionario se compone de 165 personas, elegidas por medio del programa STATS y una muestra estratificada por medio de una formula cuantitativa. Los encuestados han sido seleccionados aleatoriamente.

La sistematización de los resultados del estudio se obtendrá por medio de la revisión bibliográfica y de conocimiento teórico, datos obtenidos a través del instrumento de recolección, en este caso un cuestionario el cual comprende 2 apartados, uno de revisión sociodemográfica, económica, familiar, educativa y personal, y otro 


\section{Revista Electrónica Enfermeria Actual en costa Rica}

constituido por el instrumento Maslach, que indaga sobre el agotamiento emocional, el cinismo y la autoeficacia académica.

Para determinar la presencia o no de burnout académico por medio del instrumento Maslach, se realizará una sumatoria individual y por instrumento en cada una de sus tres categorías; con tales datos se determinará el promedio y la desviación estándar, lo cual indica que cualquier dato superior al promedio -más la suma de la desviación estándar- es sinónimo de presencia del síndrome o los síntomas. Así mismo, se determinará la no presencia del mismo a partir del promedio menos la desviación estándar. Aquellos datos que se encuentren entre estas numeraciones se consideraran en riesgo.

La validación anterior se puede comparar con la realizada por Castro, David y Ortiz (2011), en la cual indican que una puntuación superior a 53 en la totalidad de los ítems del instrumento indican presencia de burnout académico, respecto del agotamiento académico se determina que puntaciones iguales y menores a 18 significan ausencia de este y puntuaciones superiores, presencia. Para el componente de cinismo se indica que puntuaciones menores o iguales a 10 muestran ausencia de cinismo y puntuaciones mayores, su presencia. Finalmente en el componente de autoeficacia académica, puntuaciones menores o iguales a 32 equivalen a ausencia de autoeficacia académica y mayores, a presencia; sin embargo, dichos autores no consideran el rubro de riesgo de presentar el síndrome.

\section{CONSIDERACIONES ÉTICAS}

En cuanto a los principios bioéticos, en el proceso investigativo se consideró los de beneficencia, no maleficencia, autonomía y justicia, lo cual motivó el diseño del consentimiento informado para la participación en el estudio.

\section{RESULTADOS}

En cuanto a la nacionalidad de los participantes, de acuerdo con los resultados el $97,6 \%$ de los estudiantes son costarricenses.

En la tabla 1, sobre presencia del síndrome de burnout académico entres los estudiantes de enfermería, se observa que la mayoría se encuentra en riesgo con un $65,4 \%$, seguido de un $18,8 \%$ que presenta el síndrome y un $15,8 \%$ que no lo presenta.

Tabla 1

Escuela de Enfermería, UCR. Distribución de estudiantes de Enfermería según presencia del síndrome de burnout académico, 2014

(Frecuencias absolutas $f i$ y relativas $h i$ )

\begin{tabular}{l|l|l}
\hline \multirow{2}{*}{ Indicador } & \multicolumn{2}{|c}{ Frecuencias } \\
\cline { 2 - 3 } & $f i$ & $h i$ \\
\hline
\end{tabular}




\begin{tabular}{|c|c|c|}
\hline \multicolumn{3}{|l|}{$\begin{array}{l}\text { Presencia de Síndrome de Burnout } \\
\text { académico. }\end{array}$} \\
\hline Sí & 31 & 18,8 \\
\hline No & 26 & 15,8 \\
\hline En Riesgo & 108 & 65,4 \\
\hline Total & 165 & 100 \\
\hline
\end{tabular}

Fuente: Resultados de instrumento de evaluación de burnout académico, 2014

Además, un porcentaje superior a $60 \%$ se encuentra en riesgo de padecer cualquiera de los 3 síntomas propios del síndrome que son agotamiento emocional, cinismo y autoeficacia académica.

En la tabla 2, sobre presencia, riesgo o no presencia del síndrome de burnout académico, se evidencia que el síndrome se presenta más en la población de primer año (32,2\%), seguida por la de quinto año $(29,1 \%)$.

Tabla 2

Escuela de Enfermería, UCR. Distribución de estudiantes de Enfermería según presencia, riesgo o no presencia del Síndrome de burnout académico en los diferentes años de carrera.

(Frecuencias absolutas $f i$ y relativas $h i$ )

\begin{tabular}{|c|c|c|c|c|c|c|}
\hline \multirow[b]{3}{*}{ Variable } & \multirow{2}{*}{\multicolumn{2}{|c|}{$\begin{array}{c}\text { Con burnout } \\
\text { Frecuencias }\end{array}$}} & \multirow{2}{*}{\multicolumn{2}{|c|}{$\begin{array}{c}\text { En riesgo } \\
\text { Frecuencias }\end{array}$}} & \multirow{2}{*}{\multicolumn{2}{|c|}{$\begin{array}{l}\text { Sin burnout } \\
\text { Frecuencias }\end{array}$}} \\
\hline & & & & & & \\
\hline & $f i$ & $h i$ & $f i$ & $h i$ & $f i$ & $h i$ \\
\hline Primer año & 10 & 32,2 & 22 & 20,4 & 9 & 34,6 \\
\hline Segundo año & 7 & 22,5 & 17 & 15,7 & 3 & 11,6 \\
\hline Tercer año & 2 & 6,5 & 24 & 22,2 & 4 & 15,4 \\
\hline Cuarto año & 3 & 9,7 & 21 & 19,5 & 9 & 34,6 \\
\hline Quinto año & 9 & 29,1 & 24 & 22,2 & 1 & 3,8 \\
\hline Total & 31 & 100 & 108 & 100 & 26 & 100 \\
\hline
\end{tabular}

Fuente: Resultados de instrumento de evaluación de burnout académico, 2014

En la tabla 3, sobre edad y sexo de la población estudiantil se observa que la mayoría pertenece al sexo femenino (un 72\%). En cuanto a la edad, la mayoría de estudiantes se encuentra entre los 21 a 25 años representado (un 52,1\%), seguido de la población de 17 a 20 años (un 40\%).

Tabla 3

Escuela de Enfermería, UCR. Distribución de estudiantes de Enfermería, según sexo y edad, 2014

(Frecuencias absolutas $f i$ y relativas $h i$ )

\begin{tabular}{|c|c|c|}
\hline \multirow[t]{2}{*}{ Variable } & \multicolumn{2}{|c|}{ Frecuencias } \\
\hline & $f i$ & $h i$ \\
\hline Sexo & & \\
\hline
\end{tabular}




\begin{tabular}{r|c|c}
\hline Hombre & 46 & 27,9 \\
\hline Mujer & 119 & 72,1 \\
\hline Total & $\mathbf{1 6 5}$ & $\mathbf{1 0 0 , 0}$ \\
\hline Edad & & 40,0 \\
\hline De 17 a 20 & 66 & 52,1 \\
\hline De 21 a 25 & 86 & 7,9 \\
\hline De 26 a 30 & 13 & $\mathbf{1 0 0 , 0}$ \\
\hline
\end{tabular}

Fuente: Resultados de instrumento de evaluación de burnout académico, 2014

En la tabla 4, sobre presencia riesgo o no presencia del síndrome de burnout académico según su sexo, se observa que son las mujeres las que más presentan el síndrome (un 22,7\%); sin embargo, son los hombres los que más riesgo tienen de presentarlo.

Tabla 4

Escuela de Enfermería, UCR. Distribución de estudiantes de enfermería por presencia de riesgo o no presencia del síndrome de burnout académico según su sexo, 2014

(Frecuencias absolutas $f i$ y relativas $h i$ )

\begin{tabular}{c|c|c|c|c}
\hline \multirow{2}{*}{ Variable } & \multicolumn{2}{|c|}{ Hombres } & \multicolumn{2}{c}{ Mujeres } \\
\cline { 2 - 5 } & \multicolumn{2}{|c|}{ Frecuencias } & $\boldsymbol{f i}$ & $\boldsymbol{h i}$ \\
\cline { 2 - 5 } & $\boldsymbol{f i}$ & $\boldsymbol{h i}$ & 27 & 22,7 \\
\hline Con burnout & 4 & 8,7 & 16 & 13,4 \\
\hline Sin burnout & 10 & 21,7 & 76 & 63,9 \\
\hline En riesgo & 32 & 69,6 & $\mathbf{1 1 9}$ & $\mathbf{1 0 0}$ \\
\hline Total & $\mathbf{4 6}$ & $\mathbf{1 0 0}$ &
\end{tabular}

Fuente: Resultados de instrumento de evaluación de burnout académico, 2014

En la tabla 5, sobre empleo de los estudiantes, se muestra que un $86,7 \%$ de la población no tiene un trabajo remunerado, mientras que quienes sí trabajan -en su mayoría- laboran en un rango entre 4 y 10 horas semanales.

Además es importante considerar que el 97,6\% de la población es costarricense, el 95,2\% son solteros(as) y tan solo el 5,5\% tiene descendencia.

Tabla 5

Escuela de Enfermería, UCR. Distribución de estudiantes de Enfermería según trabajo remunerado y horas laboradas, 2014

(Frecuencias absolutas $f i$ y relativas $h i$ )

\begin{tabular}{lr|c|c|}
\hline \multirow{2}{*}{ Variables } & \multicolumn{2}{|c}{ Frecuencias } \\
\cline { 3 - 4 } & & $\boldsymbol{f i}$ & $\boldsymbol{h i}$ \\
\hline Trabajo remunerado & & \\
\hline & Sí & 22 & 13,3 \\
\hline
\end{tabular}




\begin{tabular}{r|c|c|}
\hline No & 143 & 86,7 \\
\hline Total & $\mathbf{1 6 5}$ & $\mathbf{1 0 0}$ \\
\hline Horas de trabajo semanales & & 86,7 \\
\hline No aplica & 143 & 1,2 \\
\hline Menos de 4 horas & 2 & 5,5 \\
\hline De 4 a 10 horas & 9 & 4,2 \\
\hline De 11 a 20 horas & 7 & 1,8 \\
\hline De 21 a 30 horas & 3 & 0,6 \\
\hline De 31 a 40 horas & 1 & $\mathbf{1 0 0}$ \\
\hline Total & $\mathbf{1 6 5}$ & \\
\hline
\end{tabular}

Fuente: Resultados de instrumento de evaluación de burnout académico, 2014

En cuanto a la parte económica, el 77,6\% de la población estudiantil recibe algún tipo de beca; un 65,5\% vive en casa propia y un $32,1 \%$, en casa de alquiler.

Por último, solo el 0,6\% debe pagar algún tipo de pensión

En cuanto al tema de ingresos del hogar los datos indican que el 80,6\% recibe un salario, mientras que un $16,4 \%$ solo recibe ingresos por algún tipo de pensión.

Además, únicamente el 1,8\% recibe ingresos por programas de ayuda económica y el 4,2\% de otras fuentes.

Por otra parte, en lo relacionado a apoyo afectivo y emocional, el 88,5\% considera que cuenta con apoyo emocional; el 93,9\%, con medios para desahogarse emocionalmente. En cuanto a las relaciones intrafamiliares, el $51,1 \%$ se encuentra muy satisfecho con las relaciones familiares seguido de un $37,6 \%$ satisfecho.

El 94,5\% tiene una persona de confianza. Respecto del tema de convivencia, la mayoría de estudiantes viven con ambos padres y hermanos (37,6\%), junto con una serie de porcentajes muy reducido de convivencia con otras personas como amigos, pareja, conocidos y otros (véase anexo 4).

Respecto de la opción de cursar otra carrera, tan solo una minoría de los estudiantes de enfermería está empadronado en una segunda, tal como se expone en la tabla 6:

Tabla 6

Escuela de Enfermería, UCR. Distribución de estudiantes de Enfermería según curso de carrera alternativa, 2014 (Frecuencias absolutas $f i$ y relativas $h i$ )

\begin{tabular}{rr|r|r}
\multicolumn{2}{|c|}{ Variables } & \multicolumn{2}{|c}{ Frecuencias } \\
\cline { 3 - 4 } & & $\boldsymbol{f i}$ & $\boldsymbol{h i}$ \\
\hline \multicolumn{1}{l|}{ Cuso de carrera alternativa } & & 32 & 19,4 \\
\hline & Só & 133 & 80,6 \\
\hline Total & $\mathbf{1 6 5}$ & $\mathbf{1 0 0}$ \\
\hline
\end{tabular}

Fuente: Resultados de instrumento de evaluación de burnout académico, 2014 


\section{Revista Electrónica Enfermeria Actual en costa Rica}

\section{www.revenf.ucr.ac.cr}

Acerca del tema anterior, un $43,8 \%$ de los estudiantes que cursan otra carrera consideran que ese factor les afecta en su desempeño académico en contraposición con el $56,2 \%$ que considera que no le afecta.

En torno al burnout, evidencia una serie de sintomatologías de diversas índoles: en cuanto a síntomas conductuales, la población estudiada indicó que los principales son irritabilidad (62,4\%) e inquietud (49,10\%).

Por otro lado, entre la población con burnout académico, se manifiestan distintos síntomas conductuales como irritabilidad (87,1\%), nerviosismo $(58,1 \%)$, inquietud $(64,5 \%)$, sentimientos de impotencia $(64,5 \%)$, sentimientos de vacío y soledad $(51,6 \%)$, sentimientos de fracaso $(54,8 \%)$, tristeza $(67,7 \%)$, pesimismo $(76,2 \%)$ y cambios bruscos de humor (67,7\%). La población que se encuentra en riesgo presenta principalmente irritabilidad (61\%) e inquietud (50\%). Por otra parte es importante mencionar que entre aquellos que no presentan el síndrome, el $42,3 \%$ no evidencia ninguno de los síntomas.

En cuanto a síntomas físicos, los principales son dolor de cabeza $(73,9 \%)$, dolor de espalda $(69,7 \%)$, trastornos del sueño (57\%) y cansancio crónico (49,7\%) (véase anexo 7). Sin embargo entre la población que presenta el síndrome, los principales son cansancio crónico $(83,9 \%)$, dolor de cabeza $(83,9 \%)$, dolor de espalda $(77,45)$, trastornos del sueño $(74,2 \%)$, Nauseas $(64,5 \%)$ y alergias $(54,8)$.

Respecto de quienes se encuentran en riesgo, los principales síntomas son el dolor de cabeza $(72,2 \%)$ y el de espalda $(73,1 \%)$

Por último, la población sin burnout académico también experimenta dolor de cabeza $(69,2 \%)$ y trastornos del sueño $(42,3 \%)$.

En cuanto a síntomas cognitivos, en la mayoría de la población se identificó dificultad para concentrarse (70,9\%), dificultad para prestar atención y memorizar $(65,5 \%)$ y más sensibilidad a la crítica (40\%) (véase anexo 9). En la población Con burnout académico, se detectó sensibilidad a la crítica (58,1\%), dificultad para concentrarse $(83,9 \%)$, dificultad para prestar atención y memorizar $(87,1 \%)$, autoestima decaída $(61,3 \%)$, dificultad para lograr metas $(64,5 \%)$ y dificultad para comprometerse con nuevos objetivos $(54,8 \%)$.

Entre la población en riesgo, se observan dos principales síntomas como la dificultad para prestar atención y memorizar $(65,7 \%)$ y dificultad para concentrarse $(69,4 \%)$. En la población que no lo presenta, el principal síntoma es dificultad para concentrarse $(61,5 \%)$.

En la tabla 7, sobre satisfacción con la carrera y el desempeño académico según presencia del síndrome, riesgo de aparición y no presencia del mismo podemos observar que en cuanto a la satisfacción con la carrera de enfermería la población con burnout, un 41,9\% se encuentra satisfecha, un 47,2\% de la población en riesgo en su mayoría se siente muy satisfecha y, entre la población que no sufre del síndrome, un 57,7\% se encuentra satisfecha y un 42,3\%, .muy satisfecha. Sin embargo, alguna parte de la población con el síndrome o en riesgo, presenta ciertos niveles de insatisfacción con la carrera.

Por otro lado, en cuanto a la satisfacción con el desempeño académico es posible observar que la mayoría ya sea que presente, no presente o se encuentre en riesgo de presentar el síndrome, se considera como satisfecha con 
su desempeño académico. No obstante, es importante tomar en cuenta que entre los estudiantes que presentan el síndrome, un $16,1 \%$ se considera muy insatisfecho, rubro que disminuye en la población en riesgo hasta un $1,9 \%$ y desaparece totalmente entre la población libre de este.

\section{Tabla 7}

Escuela de Enfermería, UCR. Distribución de estudiantes de Enfermería según satisfacción con la carrera y el desempeño académico, según presencia del síndrome de burnout académico, riesgo de aparición o no presencia del mismo, 2014

(Frecuencias absolutas $f i$ y relativas $h i$ )

\begin{tabular}{r|c|c|c|c|c|c}
\multicolumn{1}{c|}{ Variable } & \multicolumn{2}{|c|}{ Con Burnout } & \multicolumn{2}{c|}{ En riesgo } & \multicolumn{2}{c}{ Sin Burnout } \\
\cline { 2 - 7 } & \multicolumn{2}{|c|}{ Frecuencias } & \multicolumn{2}{c}{ Frecuencias } & \multicolumn{2}{c}{ Frecuencias } \\
\hline Satisfacción con la carrera de enfermería & $f i$ & $h i$ & $f i$ & $h i$ & $f i$ & $h i$ \\
\hline Muy satisfecho & 10 & 32,3 & 51 & 47,2 & 11 & 42,3 \\
\hline Satisfecho & 13 & 41,9 & 43 & 39,8 & 15 & 57,7 \\
\hline Más o menos satisfecho & 6 & 19,4 & 9 & 8,3 & 0 & 0 \\
\hline Insatisfecho & 1 & 3,2 & 3 & 2,8 & 0 & 0 \\
\hline Muy insatisfecho & 1 & 3,2 & 2 & 1,9 & 0 & 0 \\
\hline Tatisfactal & $\mathbf{3 1}$ & $\mathbf{1 0 0}$ & $\mathbf{1 0 8}$ & $\mathbf{1 0 0}$ & $\mathbf{2 6}$ & $\mathbf{1 0 0}$ \\
\hline & & & & & & \\
\hline Más o menos satisfecho & 7 & 22,6 & 21 & 19,4 & 7 & 19,2 \\
\hline Insatisfecho & 3 & 9,7 & 7 & 6,5 & 3 & 11,5 \\
\hline Muy insatisfecho & 5 & 16,1 & 2 & 1,9 & 0 & 0 \\
\hline Total & $\mathbf{3 1}$ & $\mathbf{1 0 0}$ & $\mathbf{1 0 8}$ & $\mathbf{1 0 0}$ & $\mathbf{2 6}$ & $\mathbf{1 0 0}$ \\
\hline
\end{tabular}

Fuente: Resultados de instrumento de evaluación de burnout académico, 2014

\section{DISCUSIÓN}

Esta temática ha sido poco estudiada, motivo por el que es de gran importancia observar los factores asociados o predisponentes a la aparición del síndrome dentro de la población estudiantil, para que de una u otra manera estos se puedan considerar para disminuirlos o encontrar técnicas que brinden herramientas a los estudiantes para generar habilidades que les ayuden a afrontar los altos niveles de estrés que surgen como resultado del proceso de enseñanza, de modo que no presenten el síndrome de burnout académico.

En primera instancia es necesario dar a conocer cuáles son los niveles de presencia del síndrome entre de la población estudiantil respecto de lo que es considerable mencionar que si bien la mayoría de la población no presenta el síndrome, sí se encuentra en riesgo de tenerlo, lo cual indica que estos jóvenes pueden estar 
presentando uno o más síntomas propios del burnout, pero que en realidad lo que presentan son altos niveles de estrés negativo que puede llevarlos al síndrome.

El siguiente porcentaje en importancia representa a la población con burnout académico (18,8\%), esto quiere decir que ellos presentan los 3 síntomas mencionados por Caballero, Abello y Palacio (2007), cinismo, agotamiento emocional y académico que, si bien son pocos, es trascendental obtener información que permita dar a conocer cuáles factores influyen en su aparición para así generar una respuesta ante ello.

También es importante considerar la presencia del síndrome según la presencia del síndrome, acerca de lo que se observa que los años de carrera mayormente afectados son los de primer y quinto año de carrera, en este primer caso, la presencia del síndrome puede estar relacionado con el cambio que sufren los estudiantes al pasar de ser alumnos de secundaria a universitarios, ya que, como mencionan Cova, Alvial, Aro, Bonifetti, Hernández, y Rodríguez (2007), al estar sometidos a presiones mayores ante los exámenes, falta de apoyo familiar, entre otros factores, aumenta la sintomatología ansiosa y depresiva del estudiantado, que también pueden aumentar por factores económicos y relacionales que -en muchos casos- cambian drásticamente .

Además, los estudiantes de último año son probablemente los que más situaciones estresantes pueden presentar, ya que se encuentran a pocos pasos de culminar su carrera, pero para ello deberán enfrentar situaciones estresantes como sus últimas prácticas clínicas y la elaboración de los proyectos finales de graduación que los exponen a mayores presiones académicas.

Por otra parte, en cuanto al sexo, las mujeres son las que principalmente estudian enfermería, esto se ha dado históricamente, puesto que puesto que esta tiene su origen en el cuidado materno de infantes y ha coexistido con este tipo de cuidado desde los tiempos más remotos, lo cual ha perpetuado la idea de que la enfermería solo podía ser ejecutada por mujeres (Donahue, 1988); es decir, hay una arraigada feminización de la enfermería que si bien ha ido disminuyendo paulatinamente, aún se manifiesta. Así mismo se considera que son las mujeres las que se encuentran más predispuestas a padecer del síndrome de burnout académico ya que ante situaciones de estrés son estas las que tienen mayor incidencia de sufrir de trastornos interiorizantes, tales como depresión (Martínez, Piqueras y Cándido, s.f.) lo cual también concuerda con lo que menciona Caballero, et al. (2010), quienes indican que son las mujeres las que sufren de este síndrome con mayor frecuencia. Lo anterior se ve manifestado a través del 22,7\% de mujeres que presentan el síndrome en contraposición con el 8,7\% de hombres. Si bien, los hombres presentan una mayor presencia de riesgo de presentar el síndrome es importante considerar que tan solo podría indicar altos niveles de distrés.

En lo que toca a aspectos relacionados con nacionalidad, estado civil y cantidad de hijos, no tienen gran influencia en los efectos del burnout académico sobre esta población.

También es de considerar que un gran porcentaje de los estudiantes se encuentran en el periodo de vida donde se da por terminada la adolescencia y se ingresa a la adultez joven, debido a que "la adolescencia inicia un poco después de los 10 años y concluye un poco antes de los 20" (Feldman, 2007, p.390); por tanto, lo anterior significa que un $40 \%$ de la población aún es adolescente, etapa en la que algunos podrían aun encontrarse en la búsqueda de identidad o moratoria, lo cual les podría crear insatisfacciones con la carrera o inseguridad ante sus decisiones. 
Respecto del porqué de sus jornadas laborales, hay que destacar que los horarios para matricular bloques completos de la carrera de Licenciatura en Enfermería de la Universidad de Costa Rica, en su mayoría no permiten laborar en empleos de tiempo completo, dato aportado por el 86,7\% de estudiantes que únicamente se dedica a estudiar; sin embargo, un porcentaje considerable (13,3\%) labora y, lo más importante, aproximadamente un 6,6\% lo hace por más de 11 horas semanales, lo cual redunda en menor tiempo para los estudios y el esparcimiento personal y en que, a largo plazo, se convierta en un factor estresante.

En cuanto al aspecto de ingresos económicos, la mayoría de estudiantes de enfermería reciben algún tipo de beca, tema que se relaciona con la premisa de que los ingresos y la economía de un hogar pueden considerarse como un determinante social, tal como agregan Wilkinson y Marmot, quienes mencionan que

Las circunstancias sociales y económicas deficientes afectan la salud durante la vida. Las personas que están en los estratos sociales más bajos por lo general tienen el doble de riesgo de sufrir enfermedades... las desventajas son de diversos tipos y pueden ser absolutas y relativas. Algunos ejemplos son tener un patrimonio familiar pequeño, una educación más deficiente durante la adolescencia, estar atrapado en un trabajo sin futuro o tener empleo inseguro, habitar en viviendas en malas condiciones y tratar de criar una familia en circunstancias difíciles. (2003, p.7-8)

El recibir una beca socioeconómica indica que -en su mayoría- los estudiantes no pertenecen a altos estratos sociales, sin embargo al analizar la totalidad de los datos pareciera ser que el factor económico no influye de manera significativa en la aparición del síndrome. Al respecto, se debe tomar en cuenta que "los estudiantes al igual que cualquier trabajador, mantienen una relación de compensación directa e indirecta con la institución universitaria, evidenciada en apoyos económicos, becas, reconocimientos o premios" (Caballero, et al., 2010, p. 133).

Es importante mencionar que el síndrome de burnout se ve evidenciado por un inicio de agotamiento emocional, puesto que se caracteriza por un "complejo estado afectivo, cognitivo y negativo, que se determina por el sentimiento que tienen las personas de sentirse amenazados y la impotencia para poder enfrentar a dicha amenaza, debido a que ya han agotado todos sus recursos personales y psicosociales" (Gonzales, Lacasta y Ordoñez. 2008. p. 37); por tanto, que los estudiantes tengan altos porcentajes de apoyo emocional, medios para desahogarse, personas de confianza y buenas relaciones intrafamiliares indican que existe un amplio sistema de contención emocional que funciona como un factor protector para que los niveles de burnout académico no incrementen.

En lo concerniente a la convivencia son muy pocos los estudiantes que no se encuentran acogidos durante el curso lectivo en el seno familiar. Sobre este tema, es necesario tomar en cuenta que, como menciona Savater (1999) citado por Campos (2006, p.3), "los demás nunca están de 'más', es decir, nunca son superfluos o meros impedimentos para el desarrollo de una individualidad que en realidad solo se afirma entre ellos. Para conocernos a nosotros mismos necesitamos primero ser reconocidos por nuestros semejantes"; por ende, mantener vínculos sociales, de amistad o familiares permite al individuo conocerse a sí. 
Por otro lado, en el aspecto educativo se observa que el 89,6\% de los estudiantes únicamente cursa la carrera de licenciatura en enfermería, por lo que no se considera un factor altamente influyente, a lo que se añade que tampoco afecta el desempeño de quienes cursan otra carrera.

Entre los principales síntomas conductuales de la población en general se detecta irritabilidad e inquietud, a lo que debe sumarse, en la población con burnout, nerviosismo, sentimientos de impotencia, vacío soledad y tristeza que, a la larga, puede generar la presencia de otras patologías. Sobre el tema, cabe mencionar que el síndrome de burnout tiene sus inicios en manifestaciones psicopatológicas como el aislamiento, agresividad e irritabilidad que, a su vez, se relacionan con sintomatologías somáticas como la depresión y la ansiedad: en este caso, se observa que un porcentaje importante de estudiantes presenta tales padecimientos que puede llegar a afectar la estructura familiar y personal, lo cual se puede ver reflejado en la calidad y desempeño del trabajo realizado por los estudiantes (Pérez, 2006).

Además, es fundamental destacar que entre la población sin burnout académico, un 42,3\% presenta ninguno de los síntomas conductuales, lo cual puede demostrar que estos síntomas surgen ante la presencia de estres y se agudizan en la presencia del síndrome.

En cuanto a los síntomas físicos, para Gonzales, Lacasta y Ordoñez (2008), estos surgen de una alta activación fisiológica provocada por los altos niveles de estrés mantenido en el tiempo que redundan en disfunciones fisiológicas y somáticas.

Entre las principales manifestaciones físicas, se puede mencionar problemas a nivel de sistema nervioso central, como dolores de cabeza y el insomnio, seguido de problemas osteomusculares, ocasionados por el uso de una mala técnica de mecánica corporal. De igual forma, la población con el síndrome también se ve afectada por problemas inmunológicos que aumentan la frecuencia de alergias, mientras que los síntomas físicos disminuyen ante el riesgo o la no presencia del síndrome de Burnout.

Otros síntomas importantes en este síndrome son los de origen cognitivo que según las características de la población que se estudia, pueden ser muy influyentes en el desempeño académico de los estudiantes. Si bien la teoría no indica cuál es el síntoma más común, puesto que ello depende de cada población, sí se evidencia una dificultad para concentrarse en muchos de ellos, sin olvidar que conforme aumentan los niveles de estrés del individuo aparecen problemas para prestar atención y memorizar, seguidos de otros síntomas hasta llegar a presentar el síndrome. Lo anterior se relaciona directamente con el gran esfuerzo cognitivo que aparentemente deben realizar los estudiantes de esta carrera y que afecta en gran medida aspectos de la vida diaria como el manejo del autoestima y la dificultad para lograr metas personales y cumplir objetivos propuestos que, a corto plazo, podrían ser factores de riesgo para el desempeño laboral, ya que repercuten en la calidad de trabajo en un mundo globalizado que exige competencia y en el que obtener resultados satisfactorios es importante para la motivación personal, por tanto, es necesario prepararse para enfrentar resultados favorables pero también problemas de relaciones, autodesvalorización e impotencia y fracasos profesionales que pueden llevar a mayores situaciones de estrés de las que ya se arrastran desde el periodo universitario (Alberti, De Mello, Chaveiro, y Araujo, 2009). 


\section{Revista Electrónica Enfermeria Actual en costa Rica}

Otro aspecto por considerar es la satisfacción académica, debido a que si no hay satisfacción con la carrera que se cursa puede provocar que la persona no se adapte a eventos estresantes, lo que puede generar sensaciones de no poder dar más de sí que propicien en las personas una respuesta negativa, de autocrítica desvalorizada y pérdida del interés (Caballero, Abello y Palacio, 2007).

Se debe tomar en cuenta que la mayoría de la población se considera como satisfecha con la carrera y el desempeño académico, no obstante, hay que considerar que los niveles de insatisfacción aumentan ante la presencia del síndrome de burnout académico. Respecto de lo mencionado, Caballero, Abello y Palacio (2007, p. 106) indican que

Existe relación significativa y positiva entre la autoeficacia académica con la satisfacción frente a los estudios, lo cual pone en evidencia que los alumnos que se perciben autoeficaces en sus actividades académicas muestran mayor coincidencia entre la percepción que tiene el estudiante del contexto educativo y la importancia que este le da a la universidad, a sus estudios, a la carrera y al promedio académico.

Partiendo de lo anterior, se considera que la satisfacción con el desempeño académico influye en el nivel de satisfacción en la carrera: ambos rubros deben ser tomados en cuenta, puesto la tendencia es que a mayor insatisfacción, mayor posibilidad de padecer el síndrome, a lo que se debe agregar otros aspectos propios de la población de estudiantes de enfermería como si esta carrera fue la de primera opción de ingreso a los estudios universitarios o si fue la de segunda opción, elementos que pueden provocar la aparición del síndrome de burnout.

\section{CONCLUSIONES}

De esta investigación se derivan conclusiones muy relevantes en cuanto a los aspectos sociodemográficos, como a los propios del síndrome de burnout.

En el aspecto social, la mayoría de estudiantes participantes de la investigación son del sexo femenino, de entre los 21 y 25 años de edad, quienes se encuentran principalmente en estado de soltería y sin hijos (as): este grupo representa a los más afectados por el síndrome.

Por otro lado, en cuanto al aspecto educativo, se observó que la mayoría de la población se encuentra satisfecha con la carrera de Enfermería y su desempeño, además de que se identificó que la satisfacción en estas variables es de gran importancia en la aparición del síndrome. Por otra parte, es fundamental considerar que hay un porcentaje significativo de estudiantes que cursan una carrera alternativa lo que puede aumentar su carga académica, aunque la percepción sobre si ello afecta en el desempeño académico no se puede determinar del todo. En cuanto al tiempo dedicado al estudio, la mayoría emplea 21 horas semanales o más, lo que demuestra que la dedicación académica es bastante alta.

También se observó que una minoría de la población de Enfermería posee un trabajo remunerado de al menos 4 a 10 horas semanales. En este aspecto es importante también mencionar que la gran mayoría de estudiantes poseen beca socioeconómica de la Universidad de Costa Rica, no han adquirido deudas, viven en casa propia y además, no poseen pensión, lo que indica que lo económico no es un factor altamente influyente en la aparición del síndrome en la mayoría de ellos. 


\section{Revista Electrónica Enfermeria Actual en costa Rica}

Por otro lado, en lo referente al tema de la salud, es indispensable mencionar que la mayoría de la población presenta síntomas físicos como dolores de cabeza, trastornos del sueño y problemas osteomusculares. Además de síntomas conductuales como irritabilidad, nerviosismo, sentimientos de vacío y soledad, y síntomas cognoscitivos, como dificultad para concentrarse y mayor sensibilidad a la crítica que a la larga pueden abrir el camino a otros padecimientos tanto físicos como psiquiátricos. Lo anterior es preocupante, dado que al ser enfermería una carrera basada en la práctica clínica, en donde los y las estudiantes se ven sometidos a esfuerzos tanto físicos, como emocionales y conductuales, los síntomas relacionados con los aspectos mencionados se presentan en un número significativo de la población, lo que también puede afectar su desempeño académico en gran medida.

Por otro lado, respecto a las variables propias del síndrome de burnout académico, la población que compone la carrera de Enfermería de la Universidad Costa Rica muestra en esta investigación una importante cantidad de estudiantes con Síndrome de Burnout Académico, sin embargo, lo más preocupante es que un 65\% de la población se encuentra en riesgo de presentarlo y que el aumento de estos casos se da muchas veces en el último año de carrera, lo que podría estimar que muchos tienen la posibilidad de padecerlo en los meses próximos a la culminación de carrera.

Cabe recalcar que son muchos y muy variables los aspectos que pueden contribuir a la aparición de este síndrome. No obstante, si bien los factores son diversos e influyen en mayor o menor medida, es la satisfacción académica y con la carrera de enfermería propiamente, la que interviene más en su aparición, lo cual origina una relación de a mayor insatisfacción, mayor riesgo de padecer el síndrome, lo cual concuerda con otras investigaciones anteriores.

\section{Conflicto de intereses.}

Las autoras declaran que no existe conflicto de intereses para el desarrollo de esta investigación.

\section{REFERENCIAS BIBLIOGRAFICAS}

Alberti, G., De Mello, I., Chaveiro, E. y Araujo, A. (2009). Autoestima y el trabajo docente: un estudio con profesores y estudiantes de Química de Mato Grosso, Brasil. Revista de la Sociedad Química del Perú. 75 (4). Perú: Lima. Extraído de http://www.scielo.org.pe/scielo.php?script=sci_arttext\&pid=S1810$\underline{634 X 2009000400013}$

Arango, S.; Castaño, J.; Henao, C.; Jiménez, D.; López, A. y Páez, M. (2010). Síndrome de burnout y factores asociados en estudiantes de I a X semestre de la Facultad de Medicina de la Universidad de Manizales (Colombia).Recuperado de http://bases.bireme.br/cgibin/wxislind.exe/iah/online/?IsisScript=iah/iah.xis\&src=google\&base=ADOLEC \&lang=p\&nextAction=lnk\&exprSearch=593098\&indexSearch=ID 
Barco, V.; Miranda, Y.; Herrera, D. y Caridad, Z. (2008). El Síndrome de Burnout en enfermeros básicos del segundo año de la carrera. Revista Scielo. 42 (1).Colombia: Bogotá. Recuperado de:

http://www.scielo.org.co/scielo.php?script=sci arttext\&pid=S0120-05342010000100012

Bosqued, M. (2008). Quemados: El síndrome de Burnout: ¿Qué es y cómo superarlo? Ediciones Paidós Ibérica, S.A. España.

Caballero, C., Abello, R. y Palacio, J. (2007). Relación del Burnout y el rendimiento académico con la satisfacción frente a los estudios de estudiantes universitarios. Avances en psicología latinoamericana. 25(2). 98-111.ISSN1794-4724

Caballero, C.; Hererich, C. y Palacio, J. (2010). El Burnout académico: delimitación del síndrome y factores asociados con su aparición. Revista Scielo. 24 (3-4). Cuba: Habana. Recuperado de http://scielo.sld.cu/scielo.php?script=sci arttext\&pid=S0864-03192008000300005

Campos, A. (2006). Importancia de las relaciones interpersonales en los cuidados de enfermería. .Universidad del Valle de México, 8(9). Recuperado de http://www.uvmnet.edu/investigacion/episteme/numero8y906/enfoque/a_relaciones.asp

Castro, C., David, O. y Ortiz, L. (2011). Síndrome de Burnout en estudiantes de pregrado de la universidad de Nariño. Revista electrónica de Psicología Iztacala. 14(4). Recuperado de www.iztacala.unam.mx/carreras/psicologia/psiclin

Cova, F., Alvial, W., Aro, M., Bonifetti, A., Hernández, M. y Rodríguez, C. (2007). Problemas de Salud Mental en Estudiantes de la Universidad de Concepción. Revista Terapia Psicológica. 25 (2). Chile: Santiago. Extraído de http:/www.scielo.cl/scielo.php?script=sci arttext\&pid=S0718-48082007000200001\#z

Donahue, P. (1988). Historia de la Enfermería. EE. UU: Ediciones DOYMA

Escolano, J. (2008). Autoeficacia Académica en Estudiantes de Psicología. Recuperado de http://regulated.investigacionpsicopedagogica.com/aplicaciones/RevistaEscolar/FHCCEE/espannol/leerArticulo.php?3

Feldman, R. (2007). Desarrollo Psicológico a través de la vida. México: Prentice-Hall.

Gomero, R., Palomino, J., Ruíz, F. y Llap, C. (2005). El Síndrome de Burnout en personal sanitario de los hospitales de la empresa minera de Southern Perú Copper Corporation: estudio Piloto. Revista Médica Herediana. 16 (4). Perú. Extraído de http://www.scielo.org.pe/scielo.php?pid=S1018$130 \mathrm{X} 2005000400002 \&$ script $=$ sci_arttext

Gonzales, M., Lacasta, M. y Ordoñez, A. (2008). El Síndrome de Agotamiento Profesional en Oncología. Editorial Médica Panamericana. S.A. España. 
Hernández, R., Fernández, C. y Baptista, P. (2010). (5 ed). Metodología de la investigación. México: Mc Graw Hill/ Interamericana editores, S.A.

Jácome, S.; Hederich, C.; Ordoñez, J.; Dávila, L.; González, O.; Palacio, J.; Romero, L.; Silva, M. Torrado, O. (2010). Salud Integral, desarrollo humano y calidad de vida. II Congreso de actualización en salud y II semana de psicología. Universidad del Magdalena. Recuperado de http://congresosalud.unimagdalena.edu.co/archivos/II_Congreso_Actualizacion.pdf\#page=37

León, G. (2013). ¿Puede el burnout afectar al estudiantado universitario? UNED, (4)1, pp. 130 - 147. Recuperado de http://web.uned.ac.cr/revistas/index.php/caes/article/view/134/157

Llaneza, J. (2009).Ergonomía y psicosociología aplicada. Manual para la formación del especialista. Lex Nova S.A. España.

López, N y Cerezo, S. (2011). Cuidados Paliativos en El Paciente Oncológico. Editorial Visión Libros. España: Madrid. Recuperado de http://books.google.co.cr/books?id=HQwhohdKrPMC\&printsec=frontcover\&dq=Cuidados + Paliativos + en + El+Paciente+Oncol\%C3\%B3gico\&hl=es\&sa $=$ X\&ei $=\mathrm{I} 9 \mathrm{EaUvyAE}-$ OR2QXgtIGoCw\&ved=0CD8Q6AEwAQ\#v=onepage\&q\&f=false

Martínez, A.; Piqueras, J. y Cándido, J. (S. F.). Relaciones entre Inteligencia Emocional y Estrategias de Afrontamiento ante el Estrés. Clínica Neuropsicológica Mayor, Alcantarilla. Departamento de Psicología de la Salud, Universidad Miguel Hernández. Recuperado de http://reme.uji.es/articulos/numero37/article6/article6.pdf

Oliveira, R.; Aquino, R. y Goncalves, S. (2012). Síndrome de burnout en el último año académico de pregrado de enfermería. Revista Acta Paul de enfermería. 25 (2). Brasil. Recuperado 2013 de:

http://www.scielo.br/scielo.php?script=sci_arttext\&pid=S0103-

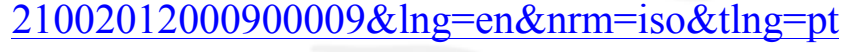

Pérez, E. (2006). Síndrome de burnout como factor de riesgo de depresión en médicos residentes. Revista Medigraphic. 22 (6). México. Extraído de http://www.medigraphic.com/pdfs/medintmex/mim2006/mim064f.pdf

Polo, A.; Hernández, J. y Poza, C. (S. F.). Evaluación del estrés académico en estudiantes universitarios. España: Madrid. Recuperado de http://www.unileon.es/estudiantes/atencion_universitario/articulo.pdf

Preciado M. y Vázquez, M. (2009). Perfil de estrés y síndrome de burnout en estudiantes mexicanos de odontología de una universidad pública. Revista chilena de neuro-psiquiatría. 48 (1). Recuperado de http://www.scielo.cl/scielo.php?script=sci_arttext\&pid=S0717-92272010000200002 


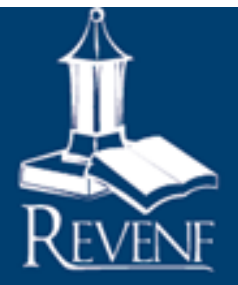

Regina, V.; Cardoso, M. y Oliveira, M. (2010). Burnout y estrés en enfermeros de un hospital universitario de alta complejidad. Revista Latinoamericana de enfermería. 18 (6). Brasil: Barao Geraldo. Recuperado de: http://www.scielo.br/pdf/rlae/v18n6/es_07.pdf

Ríos, I., Carrillo, C., Sabuco, E., (2012). Resiliencia y Síndrome de Burnout en estudiantes de enfermería y su relación con variables sociodemográficas y de relación interpersonal. International Journal of Psychological Research, 5(1), 88- 95. Recuperado de http://dialnet.unirioja.es/servlet/articulo?codigo $=3974537$

Santes, M., Meléndez, S., Martínez, N., Ramos, I., Preciado, M. y Pando, M. (2009). La salud mental y predisposición a síndrome de Burnout en estudiantes de enfermería. Revista Chilena de Salud Pública. 13(1):23-29. Recuperado de http://www.cuadernosjudaicos.uchile.cl/index.php/RCSP/article/viewFile/656/553

Wilkinson, R. y Marmot, M. (2003). Determinantes sociales de la salud: los hechos irrefutables. Organización Mundial de la salud. 